\title{
Deferred anastomosis in severe secondary peritonitis using a temporary intestinal shunt - case series
}

\author{
Ignacio Rey-Simó, Alejandra García-Novoa, Jessica Correa-Marín, Alden Pool Gómez-Alférez
}

\section{CASE REPORT}

\begin{abstract}
Introduction: As part of the damage control surgery concept applied to the treatment of sepsis due to severe secondary peritonitis following intestinal perforation and/or in high-risk patients we describe the novel "temporary intestinal shunt" (TIS) technique with delayed intestinal anastomosis, as an alternative to primary anastomosis or enterostomy.

Material and Methods: We present three patients in whom urgent laparotomy was performed due to intestinal perforation, with intestinal resection and TIS.
\end{abstract}

Case reports:

1) Case 1: A 39-year-old male presented with acute myeloid leukemia M3 and generalized peritonitis, sigmoid colon perforation and secondary jejunal loop involvement. In view of these findings, we performed $10 \mathrm{~cm}$ jejunal resection with TIS placement, sigmoid colon resection, and negative pressure therapy (NPT). Reoperation after 48 hours showed no evidence of peritonitis, so a manual jejuno-jejunal anastomosis and terminal colostomy were performed.

2) Case 2: A 65-year-old woman treated with corticosteroids presented with a pneumoperitoneum secondary to a road traffic accident. Urgent laparotomy revealed a $2 \mathrm{~cm}$ jejunal perforation. Resection of the jejunal segment and TIS with NPT was performed. Exploration of the peritoneal cavity 96 hours later showed clinical improvement and a jejuno-ileal anastomosis was performed.

3) Case 3: A 73-year-old male was admitted due to intestinal subocclusion. Clinical deterioration occurred rapidly and we performed an urgent laparotomy diagnosing jejunal perforation secondary to torsion and ischemia of the affected loop, and generalized peritonitis. Intestinal resection and TIS with NPT placement were thus decided. Anastomosis and closure of the abdominal appendage were deferred until 96 hours after the first surgery.

Conclusion: Although the evidence we present is limited, we believe TIS to be an additional tool in damage control surgery. This staged management strategy allows definitive reconstruction with the patient in a more favorable physiological condition.

Keywords - temporary intestinal shunt, deferred anastomosis, severe secondary peritonitis, sepsis, negative pressure wound therapy

Manuscript received 12.11.2019; revised 23.03.2020. This work did not receive any financial support.

Author affiliations: General Surgeon; Department of General and Digestive Surgery, Hospital Universitario de A Coruña, Spain, (IRS, AGN); General Surgeon; Hospital Universitario del Valle, Cali, Colombia, (JCM, APGA)

*Correspondence to: Ignacio Rey-Simó: ireysimo@gmail.com

\section{INTRODUCTION}

Q EVERE sepsis secondary to intrabdominal infection has $\checkmark$ bad prognosis with mortality rate that could exceed $30 \%, \frac{1+3}{1+3}$ particularly in patients with intestinal perforation. 4 The treatment of severe sepsis includes source control, restoration of gastrointestinal function, systemic antimicrobial therapy and support of organ function. A great percentage of these patients will need more than one intervention to control the source of infection. Various strategies have been proposed as abbreviated laparotomy, planned reoperation and recently, damage control surgery (DCS), in which an early temporizing operation is performed, leaving the abdomen open, followed by a planned reoperation with the definitive repair. The damage control approach allows abbreviated surgery for temporary control of the source of insult by the means of the "open abdomen" technique. Leaving the abdomen open allows for the patient to be transferred to an ICU for the restoration of homeostasis followed by a return to the operation theatre $1-2$ days later, when hemodynamic parameters have improved ( $\mathrm{pH}$, blood pressure, diuresis), for new surgical management with the definitive repair of organ defects 5

Any intestinal anastomosis has a certain risk of leakage, and although in small bowel anastomosis it is lower than in the colon, they can appear in $0.3 \%$ to $5.5 \%$ of cases, increasing the mortality up to three times.$^{7}$ Peritonitis, hypoxemia, metabolic acidosis and edema secondary to shock are intestinal leakage symptoms, particularly in an immunologically compromised patient $\frac{8}{6}$

As part of the concept of DCS9, 10 applied to the treatment of sepsis in the course of severe secondary peritonitis due to intestinal perforation in a high-risk patient (immunosuppressed, malnourished, elderly, SIRS, etc.), we describe a novel technique of "temporal intestinal shunt" (TIS) with delayed intestinal anastomosis as an alternative to the primary anastomosis or enterostomy creation.

\section{MATERIAL AND METHODS}

We present 3 patients operated at Coruña Hospital by the same surgeon. Urgent laparotomy was performed in all cases due to intestinal perforation followed by intestinal resection and TIS. In all cases, the decision of a delayed anastomosis was taken into account during the surgical procedure, assessing the patient's overall conditions $(\mathrm{pH}$, diuresis, and the need 
Table I

Clinical characteristics OF THE PATIENTS

\begin{tabular}{llll}
\hline & Case 1 & Case 2 & Case 3 \\
\hline Age (years) & 39 & 65 & 73 \\
Sex & Male & Female & Male \\
Disease history & Acute myeloid leukemia & Rheumatoid arthritis & None \\
Need for vasopressors in operating room & No & Yes & No \\
Intraoperative pH & 7.16 & 7.30 & 7.28 \\
Intraoperative lactic acid & 4 & 5 & 3 \\
No. laparotomies & 2 & 3 & 3 \\
ICU stay (days) & 20 & 7 & 4 \\
Hospital stay (days) & 47 & 34 & 15 \\
Abdominal complications & No & Acute cholecystitis and death & No \\
\hline
\end{tabular}

for vasopressors), associated comorbidities and the severity of peritonitis.

\section{Surgical technique}

The DCS was performed by resecting the affected small intestine segment. Subsequently, we inserted a fragment of a chest tube (24 to 28 fr), joining both intestinal strands, and sutured it with two silk ligatures. We then supplied a posterior laparostomy with negative pressure therapy (NPT). To perform negative pressure therapy, we used the VIVANO ${ }^{\circledR}$ System (PAUL HARTMANN AG).

This kit contained:

1) an organ protection layer to cover abdominal content;

2) two pieces of pre-shaped foam and

3) the suction connection device. 11

We provided a standard continuous pressure aspiration therapy between -80 and $-120 \mathrm{mmHg}$. Subsequently, the aspiration system was replaced every $24-48$ hours. When the patient improved an intestinal anastomosis was performed. This allowed us to conduct the surgery when local peritoneal or systemic conditions were more favorable (Fig. 1). If during the surgical review peritonitis persisted, another round of NPT was performed with the VIVANO ${ }^{\circledR}$ Tec Pro system for an additional $24-48$ hours (according to the surgeon's critical evaluation).

\section{CASE Series}

Case 1: A 39-year-old male was admitted to the hematology department for the treatment of acute myeloid leukemia in stadium M3. He presented with total medullary aplasia, abdominal pain, fever, and generalized peritonism. An urgent abdominal computed tomography scan (CTS) showed perforated sigmoid diverticulitis. Urgent surgical laparotomy revealed generalized peritonitis, sigmoid perforation and secondary affection of jejunal loop. The patient remained hemodynamically stable during surgery with metabolic acidosis $(\mathrm{pH}=7.16)$. Therefore, $10 \mathrm{~cm}$ jejunal resection with TIS placement, sigmoid colon resection and NPT with VIVANO ${ }^{\circledR}$ were performed. After 48 hours no evidence of peritonitis was observed and manual jejuno-jejunal anastomosis with a terminal colostomy (Hartmann) and definitive closure of the abdominal wall was performed. After slow recuperation secondary to the patient's concomitant myeloid leukemia the
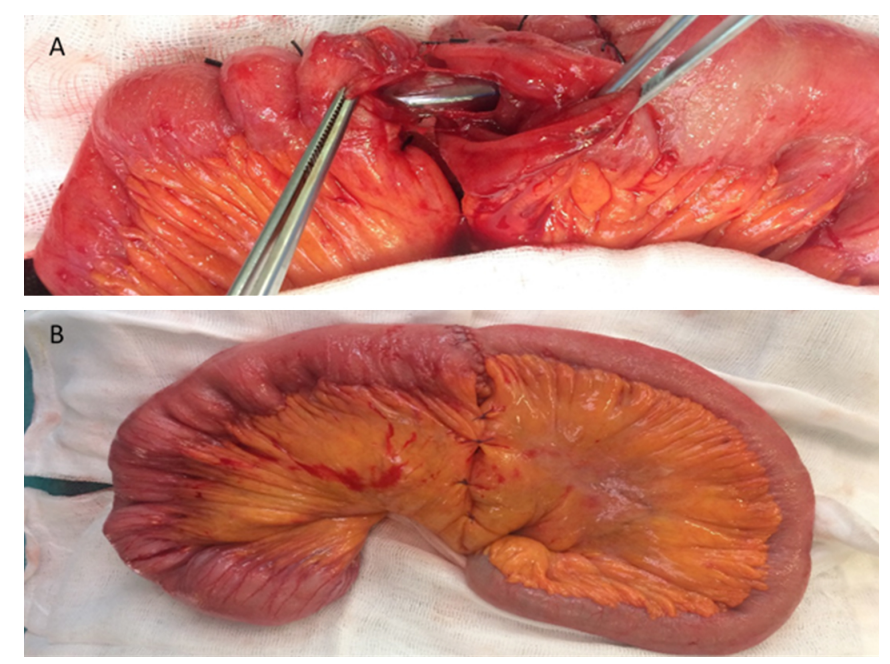

Figure 1. A. "Intestinal shunt" with thoracostomy tube $\mathrm{n}^{\mathrm{o}} 28$. B. Final intestinal anastomosis

patient could be discharged after 2 months of hospitalization and successful introduction of early oral feeding.

Case 2: A 65-year-old woman treated with corticosteroids for rheumatoid arthritis suffered a road traffic accident. She presented in the emergency room 48 hours later with vomiting and abdominal pain. CTS demonstrated pneumoperitoneum requireing an urgent laparotomy. In the operating room, we saw a $2 \mathrm{~cm}$ jejunal perforation and repaired it surgically. On the fifth postoperative day, the patient developed fever $\left(>38^{\circ} \mathrm{C}\right)$ and hypotension requireing vasopressors. Another laparotomy was performed showing evidence of diffuse peritonitis and intestinal leakage between suture points. We performed the resection of the jejunal segment followed by TIS with NPT. The cavity was revised after 48 hours with signs of active peritonitis and vacuum therapy was prolonged. After another 48 hours, a significant clinical improvement without peritonitis was observed what allowed us to perform a jejuno-ileal anastomosis. Initially, we observed a satisfactory evolution with the tolerance of the oral route nutrition but 8 days later clinical course was complicated by acute cholecystitis. Despite performing an urgent cholecystectomy, patient's general condition remained severe. 6 days after the last surgical intervention the patient died.

Case 3: A 73-year-old male was admitted to the General 
Surgery Ward with a partial small bowel obstruction. Three days after admission his clinical status deteriorated. Increasing abdominal pain and peritonism urged us to perform a laparotomy. We saw jejunal perforation secondary to a jejunal flange that was responsible for the torsion and ischemia of the affected loop and generalized peritonitis. During the intervention, the patient had significant hypotension and metabolic acidosis ( $\mathrm{pH}=7.28$ ), so that we decided to perform an intestinal resection and TIS with NPT. After 48 hours abdominal revision revealed persistent peritonitis so that the anastomosis and closure of the abdominal appendage were deferred for another 48 hours. This time the patient remained in the ICU with NPT. Successful evolution afterwards allowed us to discharge the patient in 10th postoperative day.

\section{Discussion}

Late-diagnosed perforations or anastomotic leakages in the small intestine are often catastrophic. Their incidence is higher in immunocompromised (due to corticosteroids or malignancy), malnourished, septic patients. Such leasions present with diffuse peritonitis and septic shock, dramatically increasing morbidity and mortality $(60-100 \%) ! \frac{12}{2}$ For this reason, the classical approach would be to avoid anastomosis and perform derivative enterostomata. More recently, in cases of septic shock secondary to intestinal perforation, the clinical guidelines of the World Society of Emergency Surgery 13 propose to apply the concept proposed by Stawicki et al. 17 of DCS and NPT.

DCS was initially designed for trauma patients. However, definitive surgery in a patient with severe alteration of their physiology may present adverse results, regardless of the origin of the damage. For this reason, rapid surgical management for the hemorrhage or contamination, without further major tissue injury, followed by early transfer to the intensive care unit (ICU) for resuscitation and physiological correction (acidosis, coagulopathy, and hypothermia) before performing the definitive surgery is now accepted for sepsis secondary to peritonitis. $\frac{18}{18}$ This staged management allows subsequent reconstruction of the lesions in a more favorable physiological condition.

The literature suggests DCS with affected bowel resection leaving both ends closed with mechanical sutures. There are two problems with this approach. Firstly it requires discontinuation of the intestinal transit. Secondly, it increases the possibility of intestinal leakage, which can worsen the peritonitis. In this context, our team has modified the technique using the TIS, which allows the physiological intestinal transit and decreases the pressures in the proximal cap that is often responsible for the leakage of intestinal contents through the staples and increasing the edema of the proximal end, putting the safety of the deferred anastomosis at risk.

It is evident that the appropriate selection of patients is necessary to perform this staged surgery ${ }^{6}$ The overuse of this procedure in patients who do not need it may increase the number of unnecessary surgeries, prolong the stay in the ICU and increase the risk of developing an enterocutaneous fistula. However, there is no sufficient data to establish the criteria to select patients that might have an indication to perform damage control with TIS surgery. However, in patients with generalized peritonitis and septic shock requiring vasopressors, patients with coagulopathy or acidosis, or those patients with risk factors (e.g. immunocompromised), an anastomosis would probably fail owing to the severe physiological compromise. So, in this extreme situation, it seems logical that deferring the anastomosis may result in a better outcome.

The use of a laparostomy with NPT improves the perioperative mortality rate even in non-trauma patients with abdominal symptoms. It reduces the risk of compartment syndrome, increases abdominal perfusion, decreasing the risk of renal, cardiac or pulmonary failure ${ }^{[19}$ However, it is important not to prolong the final closure of the wall, longer duration of open abdomen management and the greater number of serial abdominal explorations increase the risk of complications. Khan et al $]^{[18}$ determined that closure before the seventh day decreases postoperative morbidity, although it increases the risk of respiratory failure. Therefore, our team proposes the TIS with NPT to optimize the patient's general condition while trying not to prolong the open abdomen over 96 hours.

\section{Conclusion}

In conclusion, although the evidence we present is scarce, we believe TIS is an additional tool in damage control surgery. In the cases presented above, this technique allowed performing a delayed anastomosis, without the necessity of an enterostomy formation. Further studies will be necessary to confirm the effectiveness of this technical proposal, as well as to establish its clinical indications.

\section{REFERENCES}

[1] J. Cohen, J.-L. Vincent, N. K. Adhikari, F. R. Machado, D. C. Angus, T. Calandra, K. Jaton, S. Giulieri, J. Delaloye, S. Opal et al., "Sepsis: a roadmap for future research," The Lancet infectious diseases, vol. 15, no. 5, pp. 581-614, 2015.

[2] C. Fleischmann, A. Scherag, N. K. Adhikari, C. S. Hartog, T. Tsaganos, P. Schlattmann, D. C. Angus, and K. Reinhart, "Assessment of global incidence and mortality of hospital-treated sepsis. current estimates and limitations," American journal of respiratory and critical care medicine, vol. 193, no. 3, pp. 259-272, 2016.

[3] D. C. Angus and T. Van der Poll, "Severe sepsis and septic shock," $N$ Engl J Med, vol. 369, pp. 840-851, 2013.

[4] Z. Ye-Ting and T. Dao-Ming, "Systemic inflammatory response syndrome (sirs) and the pattern and risk of sepsis following gastrointestinal perforation," Medical science monitor: international medical journal of experimental and clinical research, vol. 24, p. 3888, 2018.

[5] A. Leppäniemi, E. J. Kimball, M. Malbrain, Z. J. Balogh, J. De Waele et al., "Management of abdominal sepsis: a paradigm shift?" Anaesthesiology intensive therapy, vol. 47, no. 4, pp. 400-408, 2015.

[6] D. Weber, C. Bendinelli, and Z. Balogh, "Damage control surgery for abdominal emergencies," British Journal of Surgery, vol. 101, no. 1, pp. e109-e118, 2014.

[7] B. C. Morse, J. P. Simpson, Y. R. Jones, B. L. Johnson, B. M. Knott, and J. A. Kotrady, "Determination of independent predictive factors for anastomotic leak: analysis of 682 intestinal anastomoses," The American Journal of Surgery, vol. 206, no. 6, pp. 950-956, 2013.

[8] M. Schein, P. N. Rogers, A. Leppäniemi, and D. Rosin, Schein's Common Sense Prevention and Management of Surgical Complications: For surgeons, residents, lawyers, and even those who never have any complications. Tfm Publishing Limited, 2013.

[9] S. S. Survivability, "Naval war publication 3-20.31," Washington, DC: Department of Defense, 1996. 
[10] J. Rezende-Neto, T. Rice, E. S. Abreu, O. Rotstein, and S. Rizoli, "Anatomical, physiological, and logistical indications for the open abdomen: a proposal for a new classification system," World Journal of Emergency Surgery, vol. 11, no. 1, p. 28, 2016.

[11] "Vivano es un innovador sistema médico para el tratamiento de heridas mediante presión negativa." Feb 2019. [Online]. Available: https://www.hartmann.info/es-es/brands/vivanoystem.

[12] S. Sharma, S. Singh, N. Makkar, A. Kumar, and M. S. Sandhu, "Assessment of severity of peritonitis using mannheim peritonitis index," Nigerian Journal of Surgery, vol. 22, no. 2, pp. 118-122, 2016.

[13] C. A. Ordóñez, Á. I. Sánchez, J. A. Pineda, M. Badiel, R. Mesa, U. Cardona, R. Arias, F. Rosso, M. Granados, M. I. Gutiérrez-Martínez et al., "Deferred primary anastomosis versus diversion in patients with severe secondary peritonitis managed with staged laparotomies," World journal of surgery, vol. 34, no. 1, pp. 169-176, 2010.

[14] R. Kafka-Ritsch, F. Birkfellner, A. Perathoner, H. Raab, H. Nehoda, J. Pratschke, and M. Zitt, "Damage control surgery with abdominal vacuum and delayed bowel reconstruction in patients with perforated diverticulitis hinchey iii/iv," Journal of Gastrointestinal Surgery, vol. 16, no. 10, pp. 1915-1922, 2012.
[15] A. W. Kirkpatrick, D. J. Roberts, P. D. Faris, C. G. Ball, P. Kubes, C. Tiruta, Z. Xiao, J. K. Holodinsky, P. B. McBeth, C. J. Doig et al., "Active negative pressure peritoneal therapy after abbreviated laparotomy: the intraperitoneal vacuum randomized controlled trial," Annals of surgery, vol. 262, no. 1, p. 38, 2015.

[16] M. Sartelli, F. Catena, F. M. Abu-Zidan, L. Ansaloni, W. L. Biffl, M. A. Boermeester, M. Ceresoli, O. Chiara, F. Coccolini, J. J. De Waele et al., "Management of intra-abdominal infections: recommendations by the wses 2016 consensus conference," World Journal of Emergency Surgery, vol. 12, no. 1, p. 22, 2017.

[17] S. P. Stawicki, A. Brooks, T. Bilski, D. Scaff, R. Gupta, C. W. Schwab, and V. H. Gracias, "The concept of damage control: extending the paradigm to emergency general surgery," Injury, vol. 39, no. 1, pp. 93-101, 2008.

[18] A. Khan, L. Hsee, S. Mathur, and I. Civil, "Damage-control laparotomy in nontrauma patients: review of indications and outcomes," Journal of Trauma and Acute Care Surgery, vol. 75, no. 3, pp. 365-368, 2013.

[19] N. Goussous, B. D. Kim, D. H. Jenkins, and M. D. Zielinski, "Factors affecting primary fascial closure of the open abdomen in the nontrauma patient," Surgery, vol. 152, no. 4, pp. 777-784, 2012. 